Bulletin UASVM Food Science and Technology 70(1)/2013, 45-52

ISSN-L 2344-2344; Print ISSN 2344-2344; Electronic ISSN 2344-5300

\title{
Comparative Fingerprint of Aromatic Herbs and Yeast Alcoholic Extracts used as Ingredients for Promen, a Prostate Preventive Nutraceutical
}

\author{
Florina CSERNATONI ${ }^{1,2)}$, Carmen SOCACIU ${ }^{1)}$, Raluca Maria POP ${ }^{2,3)}$, Floricuța \\ RANGA $^{1)}$, Florina BUNGHEZ ${ }^{1)}$, Florina ROMANCIUC ${ }^{1,2)}$ \\ ${ }^{1)}$ University of Agricultural Sciences and Veterinary Medicine, Faculty of Agriculture, 3-5 Mănăştur \\ Street, Cluj-Napoca, Romania; florina.csernatoni@gmail.com \\ ${ }^{2)}$ Center for Applied Biotechnology CCD-BIODIATEC, Proplanta Cluj-Napoca, Romania \\ ${ }^{3)}$ University of Medicine and Pharmacy "Iuliu Hatieganu" Cluj-Napoca, Victor Babes, 8, Cluj- \\ Napoca, Romania
}

\begin{abstract}
The aim of this study was to characterize and identify different bioactive compounds in plant sources and yeast powders to obtain an original nutraceutical (Promen) which has beneficial effects in prostate disease prevention.

Seven plant and fruit sources, namely nettle (Urtica dioica), green tea (Camellia sinensis), fluff with small flowers (Epilobium parviplorum), tomato (Solanum licopersicum), sea buckthorn (Hippophae rhamnoides), pumpkin (Cucurbita maxima), sunflower (Helianthus annus) and lyophilized beer yeast (Saccharomyces cerevisiae) were investigated. Methanolic extracts were prepared using $15 \%$ plant concentration and the purified fractions were analyzed using high throughput techniques like UV-VIS spectroscopy, high performance liquid chromatography coupled with photodiode array detection (HPLC-DAD) and mass spectrometry LC-QTOF -MS.

The majority of the investigated plants were rich in phenolic derivatives, polyphenols (flavonoid glucosides), while yeast was rich in aminoacids, peptides and vitamins B. The major compounds identified were: Juglone, Resveratrol, Quercetin, Epigallocatechin, Gallocatechin, Biochanin A, Isorhamnetin 3-O-glucoside 7-O-rhamnoside, Quercetin 3-O-galactoside 7-Orhamnoside, Kaempferol 3,7-O-diglucoside and $p$-Coumaroylquinic acid.

The specific biomarkers were identified for both plant extracts used as ingredients to obtain an nutraceutical Promen.

Combined UV-Vis spectroscopy, HPLC-PDA chromatography and LC-MS spectrometry are recommended as accurate, sensible and reliable tools to investigate the plants and nutraceutical fingerprints and to predict the relation between ingredients composition and their health effects.
\end{abstract}

Keywords: aromatic plants, yeast, prostate protection, UV-VIS spectroscopy, LC-MS spectrometry.

\section{INTRODUCTION}

The management of prostate cancer and its increasing incidence need novel preventive approaches like chemoprevention, by the administration of synthetic compounds or, better, using natural formula (Adhami et al., 2007).The prostate diseases generally are detected in men in their fifties or older. The prostate cancer evolution implies a considerable period of time, lifestyle changes or use of dietary or chemo preventive agents that might delay the development or onset of clinically detectable disease (Thompson et al., 2013).

The natural dietary supplements based on herbs or derived from plants or other natural nutritional agents can be used for this purpose, the epidemiologic, clinical, or basic science revealed their efficiency for prostate cancer prevention (Marshall, 2012). Moreover, recent studies showed that medicinal plants have beneficial effects on health promotion, out of side effects, as compared with synthetic drugs (Katz, 2007). 
Herbs are known for their antioxidant activity and immunomodulatory leading to anticarcinogenic effect. The main antioxidants with anticancer activity found in plants and vegetables include vitamins, carotenoids, flavonoids, polyphenols, enzymes, minerals, saponins, lignin (Behara and Dash, 2012). The main herbs and fruits known for the antoxidant and anticarcinogenic effects are nettle (Urtica dioica), green tea (Camellia sinensis) and fluff with small flowers (Epilobium parviplorum), tomato (Solanum licopersicum), sea buckthorn (Hippophae rhamnoides), pumpkin (Cucurbita maxima) sunflower (Helianthus annus) and lyophilized beer yeast (Saccharomyces cerevisiae).

The sea buckthorn berries have a good chemoprotective effect (Upendra et al., 2008) on prostate diseases because of its high content in bioactive compounds like lycopene, vitamins , $\beta$-caroten and phenolic acids and flavonoids (Novruzov, 2005).

Nettle consumption reduces the risk of prostate disease due to its antimicrobial, antiinflammatory and anti-tumor activity because it contains polyphenols, tannins, triterpenes and beta-sitosterol (Lowe and Patel, 2008). Green tea is known to have chemoprotective effects due to its high content in catechins and tannins, with antioxidant action against prostate cancer risk (Oliveira et al., 2013). Tomatoes chemoprotective effect in prostate diseases is due to their high content in carotenoids (especially lycopene and $\beta$-carotene) and minerals like selenium (Behara, 2012; Kujawski, 2009).

Fluff with small flowers is active in preventing and treating prostate adenoma and cancer due to its high content in polyphenols (Awad and Fink, 2000). Pumpkin and sunflower seeds are important for prevention of prostate diseases because their high content of phytosterols and minerals (Hernández, 2012; Alfawaz, 2004).

Lyophilized beer yeast (Saccharomyces cerevisiae) has chemoprotective effects on prostate diseases due to its high content of bioactive compounds like vitamins, mineral and aminoacids (Blagovi et al,. 2001).

The UV-Vis spectroscopy is a simple, cheap and easy-to-use technique to identify and quantify the main phytochemicals, discriminating between the lipophilic and hydrophilic phytochemicals, in relation to the polarity of the extraction solvent (Zavoi et al., 2011). Other advanced techniques for evaluation of an herbal product by its metabolomic fingerprinting can be HPLC with UV or diode-array detection (DAD) (Kammerer, 2004; Tang, 2008) as well with mass spectrometry detection.

The aim of this study was to investigate a number of medicinal plants with known beneficial effects, as ingredients for an original formula named PROMEN, a nutraceutical which can be recommended in the prevention and treatment of prostate cancer. The specific chemical biomarkers for individual plants and their possible recognition in the final product were identified by UV-Vis, HPLC-DAD and LC QTOF-MS analysis.

\section{MATERIALS AND METHODS}

\section{Plant ingredients and PROMEN preparation}

Seven types of medicinal plants and fruits from wild flora of different areas of Transylvania were numbered as follows: 1- nettle, 2- green tea, 3- fluff with small flowers, 4tomato, 5- sea buckthorn ,6- pumpkin, 7- sunflower, 8- lyophilized beer yeast and 9- final product obtained Promen. The collected plants were dried, ground and stored in a cool place $\left(10-15^{0} \mathrm{C}\right)$ with low humidity. Promen product was achieved by mixing different proportions of the seven plants were dried and ground. The weight ration was: $0.5: 0.5: 0.5: 2: 0.5: 1: 1: 4$. 


\section{Extraction of bioactive compounds}

Aliquots of $1.5 \mathrm{~g}$ of each plant powder (1-8) and Promen (9) were extracted in $8.5 \mathrm{ml}$ solvent (methanol $96 \%$ in water, acidulated with $1 \%$ hydrochloric acid. After sonication for $30 \mathrm{~min}$, centrifugation and filtration, the clear extracts were kept in deep freezer until analysis.

\section{UV-Vis spectra}

The UV-Vis spectra were recorded for each extract using a Jasco V 530 Spectrophotometer. There were identified the maxima wavelengths specific for phenolic acids (220-280 $\mathrm{nm})$, flavonoids (330-360 nm) and/or quinones (398-420 nm).

\section{Total polyphenols content}

Total polyphenols content of methanolic extracts 1-9 was determined using Folin Ciocalteu method. $2.350 \mathrm{ml}$ distilled water, $0.05 \mathrm{ml}$ sample, $0.150 \mathrm{ml}$ Folin-Ciocalteu reagent and $0.450 \mathrm{ml} \mathrm{Na} 2 \mathrm{CO} 3$ were added to the pleasant work; For the $0.05 \mathrm{ml}$ blank sample were replaced with $0.05 \mathrm{ml} 40 \%$ ethanol; After 2 hours in the dark plates were read multidetector spectrometer working at Biotek.

\section{Extraction efficiency}

To compare the yields of extraction the Extraction Factors (EF) of phenolic acids (EF-FA), flavonoids (EF-F) and quinones (EF-Q) from each plant and Promen were calculated according to the formula: $\mathrm{EF}=\mathrm{A}\left(\lambda_{\max }\right) \times \mathrm{D}$, where $\mathrm{A}\left({ }_{\lambda_{\max }}\right)$ represents the absorption values recorded for each $\lambda_{\max }$ identified in the UV-Vis spectra and D represents the dilution factor. The $\lambda_{\max }$ values used for EF-FA, EF-F and EF-Q were 280, 330 and $410 \mathrm{~nm}$, respectively.

\section{HPLC-DAD AND LC-ESI(+)QTOF-MS analysis}

All plant extracts and Promen extract were diluted (1:1) with methanol and aliquots of $5 \mu \mathrm{l}$ of each sample were subjected to two types of chromatography, HPLC coupled with photodiode array detection (HPLC-DAD) and LC-ESI (+) QTOF-MS analysis, both using a Thermo Scientific HPLC UltiMate 3000 system equipped with a quaternary pump delivery system Dionex and MS detection by a Bruker Daltonics MaXis Impact device.

The plant metabolites were separated on the Thermo Scientific Acclaim $\mathrm{C}_{18}$ column $(3 \mu \mathrm{m}, 2.1 \times 50 \mathrm{~mm})$ at $40^{\circ} \mathrm{C}$. The mobile phase consisted of $0.1 \%$ formic acid in water (A) and $0.1 \%$ formic acid in acetonitrile (B). The flow rate was set at $0.5 \mathrm{~mL} \cdot \mathrm{min}^{-1}$. The gradient elution initial conditions were $1 \% \mathrm{~B}$ with linear gradient to $15 \% \mathrm{~B}$ from 0 to $3 \mathrm{~min}$, followed by linear gradient to $50 \% \mathrm{~B}$ at $6 \mathrm{~min}$, linear gradient to $95 \% \mathrm{~B}$ at $9 \mathrm{~min}$, isocratic on $95 \% \mathrm{~B}$ for $6 \mathrm{~min}$ and then returned to initial conditions at $15 \mathrm{~min}$ and kept isocractic on $1 \% \mathrm{~B}$ for 5 min. The DAD detector was set at $270 \mathrm{~nm}$. The separated molecules were introduced directly into the mass spectrometer by electrospray. The mass range was set between $50-1000 \mathrm{~m} / \mathrm{z}$, using a nebulising gas pressure set at 2 bar, the drying gas flow at $8 \mathrm{~L} / \mathrm{min}$, the drying gas temp at $180{ }^{\circ} \mathrm{C}$. Before each separation run, a calibrant solution of sodium formate was injected. The control of the instrument and the data processing were done using TofControl 3.2 and Data Analysis 4.1 (Bruker Daltonics), respectively. 


\section{RESULTS AND DISCUSSIONS}

\section{Extracts fingerprint by UV-VIS analysis and extraction efficiency}

Tab.1

Comparative UV-VIS fingerptints of methanolic sample extracts

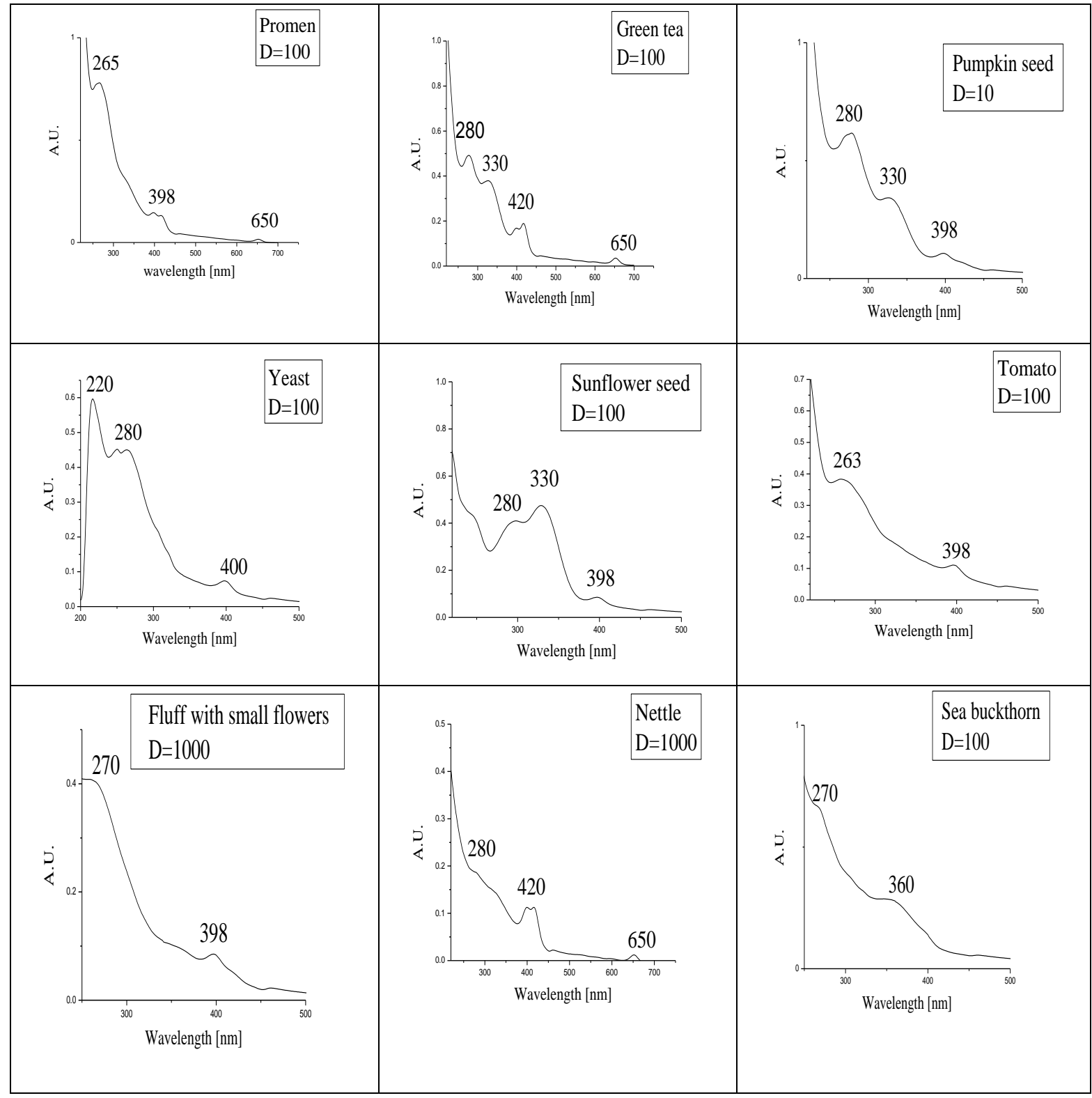

The Tab. 1 shows the comparative UV spectral fingerprints $(200-700 \mathrm{~nm})$ of the nutraceutical product Promen and its plant ingredients.

Previously we made the spectra of all ingredients, the green tea, pumpkin seed, sunflower seed and yeast extracts showed three main peaks, at 280, 330 and $400 \mathrm{~nm}$ while the Promen, tomato, fluff with small flowers, nettle and sea buckthorn had only two absorption regions at 280 and $400 \mathrm{~nm}$.

The evaluation of Extraction efficiency according to the formula presented above (see materials and methods) sowed that generally, the phenolic acids were extracted better 
than flavonoids, being maximal for fluff with small flowers and approximately 10 times smaller, compared with nettle. The nonpolar components from pumpkin seeds, sunflower seed, sea buckthorn and tomatoes were not extracted in the hydrophilic solvent, explaining the low concentrations of FA, F and Q released (for abbreviations see materials and methods). Other explanation could be that the other plants used as ingredients with high content in polar compounds contribute to the extraction efficiency in limited amount due to their 5\% contribution in the final product.

\section{Total polyphenols concentration}

The total polyphenol content identified in ingredient plants and Promen are shown in Tab. 1, being expressed in gallic acid equivalents per $100 \mathrm{ml}$ extract.

Tab. 2.

The mean values of polyphenol content for each plant and Promen

\begin{tabular}{|l|c|}
\hline \multicolumn{1}{|c|}{ Samples } & $\begin{array}{c}\text { Total polypheols } \\
\text { [mg GAE/ 100 ml extract }]\end{array}$ \\
\hline Promen & $\mathbf{7 6 . 6 1 1}$ \\
\hline Yeast & 8.843 \\
\hline Tomato & 15.381 \\
\hline Pumpkin seed & 11.342 \\
\hline Sunflower seed & 39.386 \\
\hline Nettle & 55.347 \\
\hline Fluff with small flowers & 191.210 \\
\hline Green tea & 57.846 \\
\hline Sea buckthorn & 40.225 \\
\hline
\end{tabular}

The fluff with small flowers had the highest polyphenol content being aproximatively 2.5 times higher than Promen while all the other extracts had lower phenolic concentrations.

\section{HPLC-DAD AND LC-ESI(+)QTOF-MS analysis}

Fig. 1A represents a generic HPLC-DAD chromatogram of Promen and Fig. 1B represents the TIC (Total Ion chromatograms) obtained for the same final product.

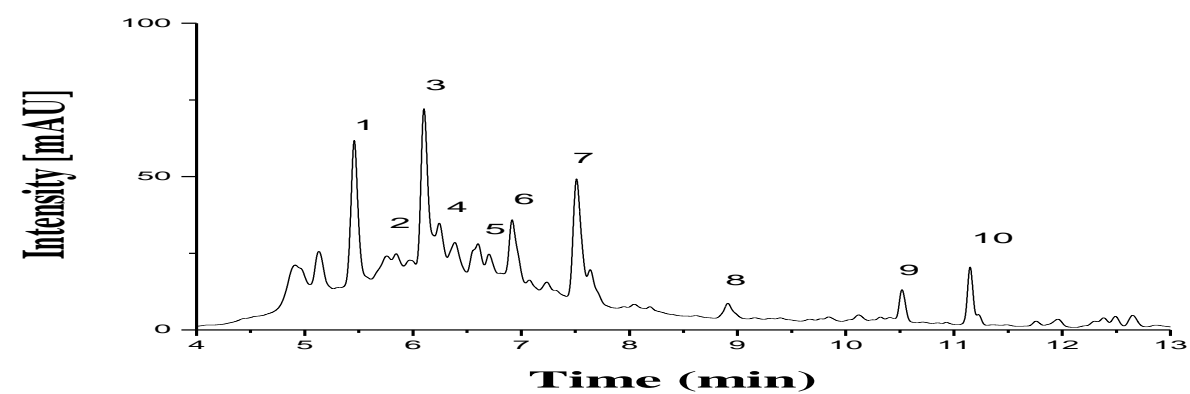

Fig. 1. A- HPLC-DAD chromatogram and compounds identified in Promen extract between 5.5-11 min. 


\section{Tab. 3.}

Main compounds identifed in Promen extract between 5.5-11

\begin{tabular}{|c|c|c|c|}
\hline Peak number & Retention time (min) & Peak number & Retention time (min) \\
\hline 1 & 5.5 & 6 & 6.9 \\
\hline 2 & 5.8 & 7 & 7.5 \\
\hline 3 & 6.1 & 8 & 8.9 \\
\hline 4 & 6.2 & 9 & 10.5 \\
\hline 5 & 6.6 & 10 & 11.1 \\
\hline
\end{tabular}

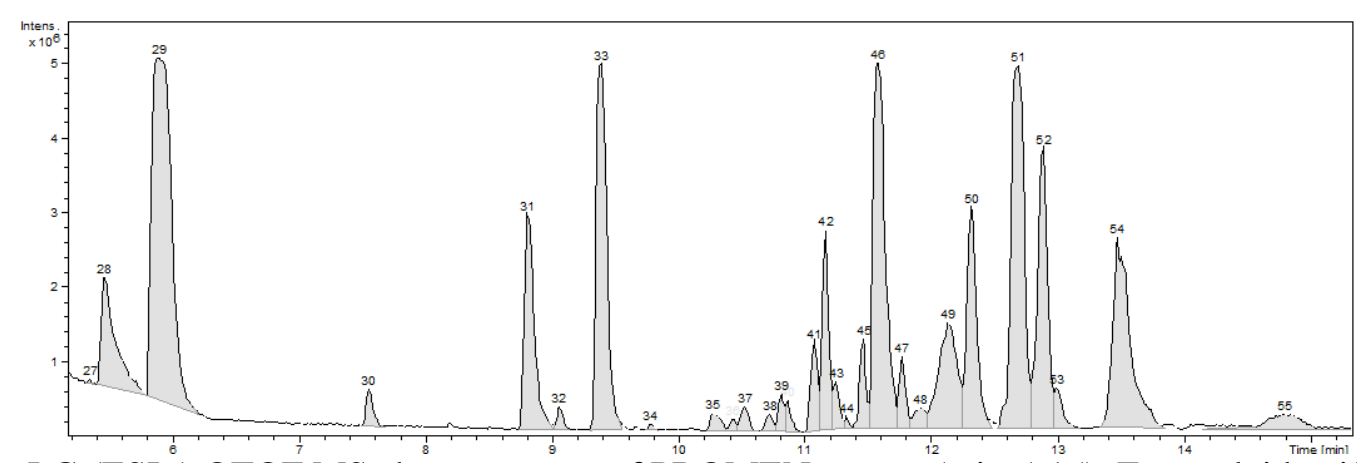

Fig. 1B. LC-(ESI+) QTOF MS chromatogram of PROMEN extract (min 5-15). For peak identification see Tab. 4

The tentative assignment of compound identification was based on their retention times, the released ions of protonated molecules $[\mathrm{M}+\mathrm{H}]^{+}$and literature data (Yanga et al., 2009; http://www.phenol-explorer.eu).

Tab. 4

Main compounds identifed in Promen extract between 5.5-14.8 min. and tentavive structure assignment based on $[\mathrm{M}+\mathrm{H}]^{+}$

\begin{tabular}{|c|c|c|c|c|}
\hline $\begin{array}{c}\text { Peak } \\
\text { number }\end{array}$ & $\begin{array}{l}\text { Retention } \\
\text { time (min) }\end{array}$ & Tentative structure assignment & $\begin{array}{c}{[\mathrm{M}+} \\
\mathrm{H}]^{+} m / z\end{array}$ & Plant ingredient \\
\hline 28 & 5.5 & Ferulic acid & 195.08 & Yeast, tomato \\
\hline 29 & 5.9 & Juglone & 175.06 & Sunflower seed \\
\hline 30 & 7.6 & Isorhamnetin & 319.13 & Sea buckthorn \\
\hline 31 & 8.8 & Resveratrol & 230.24 & Sea buckthorn, green tea \\
\hline 33 & 9.4 & Quercetin & 302.30 & Green tea, fluff with small flowers \\
\hline 34 & 9.8 & $\begin{array}{l}\text { 5,3',4'-Trihydroxy-3-methoxy-6:7- } \\
\text { methylenedioxyflavone 4'-O-glucuronide }\end{array}$ & 520.34 & Sunflower seed \\
\hline 38 & 10.7 & Caffeoylquinic acid & 353.26 & $\begin{array}{l}\text { Yeast, tomato, pumpkin seed, sunflower } \\
\text { seed, nettle }\end{array}$ \\
\hline 39 & 10.8 & Gallic acid 3-O-gallate & 324.29 & Nettle, pumpkin seed, tomato \\
\hline 41 & 11.1 & Feruloylquinic acid & 365.26 & Pumpkin seed \\
\hline 42 & 11.2 & Epigallocatechin, Gallocatechin & 306.24 & Green tea, fluff with small flowers, nettle \\
\hline 43 & 11.2 & Caffeoyl aspartic acid & 295.26 & $\begin{array}{l}\text { Sea buckthorn, pumpkin seed, sunflower } \\
\text { seed }\end{array}$ \\
\hline 44 & 11.3 & Feruloyl tartaric acid & 326.30 & Sea buckthorn \\
\hline 45 & 11.5 & Pinocembrin & 256.26 & Tomato, yeast \\
\hline 46 & 11.6 & Biochanin A & 284.28 & Pumpkin seed \\
\hline 47 & 11.8 & Apigenin 7-O-diglucuronide & 621.27 & Fluff with small flowers, nettle \\
\hline 50 & 12.3 & Isorhamnetin 3-O-glucoside 7-O-rhamnoside & 623.29 & nettle, green tea \\
\hline 51 & 12.7 & Quercetin 3-O-galactoside 7-O-rhamnoside & 607.29 & Nettle, green tea, fluff with small flowers \\
\hline 52 & 12.9 & Kaempferol 3,7-O-diglucoside & 607.29 & Nettle, green tea, fluff with small flowers \\
\hline 53 & 13 & Resveratrol 5-O-glucoside & 391.28 & Tomato, sea buckthorn \\
\hline 54 & 13.5 & p-Coumaroylquinic acid & 338.34 & All plants \\
\hline 55 & 14.8 & $\begin{array}{l}\text { Luteolin 7-O-(2-apiosyl-6-malonyl)- } \\
\text { glucoside }\end{array}$ & 664.43 & Yeast, sunflower seed \\
\hline
\end{tabular}


The majority of the investigated plants were rich in phenolic derivatives, polyphenols (flavonoid glucosides), while yeast was rich in aminoacids, peptides and vitamins B. The major compounds identified were: Juglone, Resveratrol, Quercetin, Epigallocatechin, Gallocatechin, Biochanin A, Isorhamnetin 3-O-glucoside 7-O-rhamnoside, Quercetin 3-Ogalactoside 7-O-rhamnoside, Kaempferol 3,7-O-diglucoside and $p$-Coumaroylquinic acid.

\section{CONCLUSION}

The phenolic acids were extracted better than flavonoids, being maximal for fluff with small flowers. The nonpolar components from samples were not extracted in the hydrophilic solvent, explaining the low concentrations of FA, F and Q released.

The fluff with small flowers had the highest polyphenol content being aproximatively 2.5 times higher than Promen while all the other extracts had lower phenolic concentrations.

Using LC-QTOF-MS analysis 21 specific compounds were identified in the final product Promen and in the different plant extracts used as ingredients.

The main biomarkers which differentiate the individual plants were identified and will be further used to evaluate the quality of nutraceutical product and their synergistic effect against prostate metabolic dysfunction.

Combined UV-Vis and HPLC-PDA and LC-MS chromatography can be recommended as accurate, sensible and reliable tools to investigate the plants and nutraceuticals' fingerprints and to predict the relation between the ingredients' composition and effects.

\section{REFERENCES}

1. Adhami V.M., A. Malik, N. Zaman, S. Sarfaraz, I. A. Siddiqui, D.N. Syed, F. Afaq, F.S. Pasha, M. Saleem, and H. Mukhtar (2007). Combined Inhibitory Effects of GreenTea Polyphenols and Selective Cyclooxygenase-2 Inhibitors on the Growth of Human Prostate Cancer Cells Both In vitro and In vivo. Clin Cancer Res 13:1611-1619.

2. Alfawaz M. A. (2004). Chemical composition and oil characteristics of pumpkin (Curcubita maxima) seed kernels. Food Sci \& Agric. Res. Center 129: 5-18.

3. Awad A.B. and C.S. Fink (2000). Phytosterols as anticancer dietary components: evidence and mechanism of action. J Nutr 130(9):2127-2130.

4. Behara S.V. and V. Dash (2012). Some indian vegetables used as anticancer agent. Int J Adv Pharm Biol Sci 2(4): 250-264.

5. Blagovi B., J. Rupi, M. Mesari,K. Georgiú, V. Mari (2001). Lipid Composition of Brewer's Yeast. Food technol. biotechnol. 39(3): 175-181.

6. Lowe F.C. and T. Patel (2008). Complementary and alternative medicine in urology: what we need to know. BJU International 102(4): 422-424.

7. Kammerer D., C. Achim, R. Carle and A. Schieber (2004). Polyphenol Screening of Pomace from Red and White Grape Varieties (Vitis vinifera L.) by HPLC-DAD-MS/MS. J. Agric. Food Chem., 52 (14): 4360-4367

8. Katz A.E. (2007). The holistic approach to prostate health. Integrative Medicine 6(3): 50-59.

9. Kujawski R., M. Ozarowski, N.D. Holysz, J. B. Wieczorek, A. Bogacz, M. Karasiewicz, P. L. Mikolajczak, T. B. Kozlowska, P. M. Mrozikiewicz (2009). Effect of Willow herb (Epilobium angustifolium L.) extract on gene expression of selected P450 cytochromes in rat liver - preliminary study. Herba Polonica 55(4):52-63.

10.Luis G, C. Hernández, C. Rubio, D. González-Weller, A. Gutiérrez , C. Revert, A. Hardisson (2012). Trace elements and toxic metals in intensively produced tomatoes (lycopersicon esculentum). Nutr Hosp. 27(5):1605-1609.

11.Marshall J. R. (2012). Diet and prostate cancer prevention. World J Urol 30:157-165 
12.Novruzov E.N, (2005). Biochemical characteristics of Seabuckthorn (Hippophae rhamnoides L.) Growing in Azerbaijan, in : Seabuckthorn (Hippophae L), A Multipurpose Wonder Plant. ed. V. Singh, 2:133-144.

13. Oliveira P. F., B. M. Silva, T. R. Dias, G. Tomás, N. F. Teixeira, M. G. Alves (2013). White Tea (Camellia Sinensis (L.)): Antioxidant Properties and Beneficial Health Effects. International Journal of Food Science, Nutrition and Dietetics, 2(2):1-15.

14.Tang D., L. Hui-Jun , J. Chen, G. Chao-Wei, L. Ping (2008). Rapid and simple method for screening of natural antioxidants from Chinese herb Flos Lonicerae Japonicae by DPPH-HPLC-DADTOF/MS. Journal of Separation Science 31(20): 3519-3526.

15.Thompson M.I., P.J. Goodman, C.M. Tangen, H.L. Parnes, L.M. Minasian, P.A. Godley, M.S. Luciab and L.G. Ford (2013). Long-Term Survival of Participants in the Prostate Cancer Prevention Trial. N Engl J Med 369(7): 603-610.

16.Upendra K. S, K. Sharma, N. Sharma, A. Harma, H. P. Singh, A. K. Sinha (2008). Microwave-Assisted Efficient Extraction of Different Parts of Hippophae rhamnoides for the Comparative Evaluation of Antioxidant Activity and Quantification of Its Phenolic Constituents by Reverse-Phase High-Performance Liquid hromatography (RP-HPLC). J. Agric. Food Chem., 56:374379.

17. Yanga M., J. Sunb, L. Zhiqiang, C. Guangtong, G. Shuhong, L. Xuan , J. Baohong, Y. Min , D.A. Guo (2009). Phytochemical analysis of traditional Chinese medicine using liquid chromatography coupled with mass spectrometry. Journal of Chromatography 1216(11): 2045-2062.

18.Zavoi S. , F. Fetea, F. Ranga, R. M. Pop , A. Baciu , C. Socaciu (2011). Comparative Fingerprint and Extraction Yield of Medicinal Herb Phenolics with Hepatoprotective Potential, as Determined by UV-Vis and FT-MIR Spectroscopy. Not Bot Horti Agrobo 39(2):82-89.

19.http://www.phenol-explorer.eu/ 